\title{
Meta-Analysis of Low Density Lipoprotein Receptor (LDLR) rs2228671 Polymorphism and Coronary Heart Disease
}

\author{
Huadan Ye, ${ }^{1,2}$ Qianlei Zhao, ${ }^{3}$ Yi Huang, ${ }^{1}$ Lingyan Wang, ${ }^{4}$ Haibo Liu, ${ }^{3}$ Chunming Wang, \\ Dongjun Dai, ${ }^{1}$ Leiting $\mathrm{Xu},{ }^{1}$ Meng Ye, ${ }^{2}$ and Shiwei Duan ${ }^{1}$ \\ ${ }^{1}$ Zhejiang Provincial Key Laboratory of Pathophysiology, School of Medicine, Ningbo University, Ningbo, Zhejiang 315211, China \\ ${ }^{2}$ The Affiliated Hospital, School of Medicine, Ningbo University, Ningbo, Zhejiang 315211, China \\ ${ }^{3}$ Yinzhou People's Hospital, School of Medicine, Ningbo University, Ningbo, Zhejiang 315040, China \\ ${ }^{4}$ Bank of Blood Products, Ningbo No. 2 Hospital, Ningbo, Zhejiang 315010, China
}

Correspondence should be addressed to Meng Ye; dryemeng@yahoo.com.cn and Shiwei Duan; duanshiwei@nbu.edu.cn

Received 29 January 2014; Revised 3 April 2014; Accepted 22 April 2014; Published 12 May 2014

Academic Editor: Hongwei Wang

Copyright (C) 2014 Huadan Ye et al. This is an open access article distributed under the Creative Commons Attribution License, which permits unrestricted use, distribution, and reproduction in any medium, provided the original work is properly cited.

\begin{abstract}
Low density lipoprotein receptor (LDLR) can regulate cholesterol metabolism by removing the excess low density lipoprotein cholesterol (LDL-C) in blood. Since cholesterol metabolism is often disrupted in coronary heart disease (CHD), LDLR as a candidate gene of CHD has been intensively studied. The goal of our study is to evaluate the overall contribution of $L D L R$ rs2228671 polymorphism to the risk of CHD by combining the genotyping data from multiple case-control studies. Our metaanalysis is involved with 8 case-control studies among 7588 cases and 9711 controls to test the association between $L D L R$ rs2228671 polymorphism and CHD. In addition, we performed a case-control study of $L D L R$ rs2228671 polymorphism with the risk of CHD in Chinese population. Our meta-analysis showed that rs2228671-T allele was significantly associated with a reduced risk of CHD $(P=0.0005$, odds ratio $(\mathrm{OR})=0.83$, and $95 \%$ confidence interval $(95 \% \mathrm{CI})=0.75-0.92)$. However, rs2228671-T allele frequency was rare $(1 \%)$ and was not associated with CHD in Han Chinese $(P=0.49)$, suggesting an ethnic difference of $L D L R$ rs2228671 polymorphism. Meta-analysis has established rs2228671 as a protective factor of CHD in Europeans. The lack of association in Chinese reflects an ethnic difference of this genetic variant between Chinese and European populations.
\end{abstract}

\section{Introduction}

Coronary heart disease (CHD) is a complex disease caused by an insufficient blood flow inside the coronary vessels [1]. The blockage of the arteries is often caused by the plaque accumulated in the wall of arteries. The plaque is formed by excess low density lipoprotein cholesterol (LDL-C) in blood that dramatically increases the risk of CHD [2]. Low density lipoprotein receptor (LDLR) plays a key role in the regulation of cholesterol metabolism by removing excess LDL-C in blood $[3,4]$.

CHD is caused by both environmental and genetic factors [5]. Variations of genes involved in lipoprotein and lipid metabolism are playing an important role in the susceptibility of CHD [6]. LDLR gene mutations can lead to deficiency or abnormality of LDLR in the cell membrane surface and thus disrupt lipid metabolism [4]. LDLR gene mutations are known to cause familial hypercholesterolemia (FH) [2] that is an important risk factor of CHD and other atherosclerotic diseases [7]. Recent genome-wide association studies (GWASs) showed that LDLR gene mutations were significantly associated with the abnormal blood lipid levels and $\mathrm{CHD}[8,9]$. Among the $L D L R$ polymorphisms, rs 2228671 was associated with LDL-C levels and CHD in German and British populations [10-14]. However, discrepancies were also shown in the association of $L D L R$ rs2228671 with CHD in Italians and Germans $[15,16]$.

Meta-analysis is able to combine and review the results from previous studies $[17,18]$. Meta-analysis improves the power of comprehensive statistics by pooling the data from different studies. In the present study, we performed a metaanalysis of $L D L R$ rs 2228671 polymorphism with $C H D$ among 17299 individuals in 8 studies. 


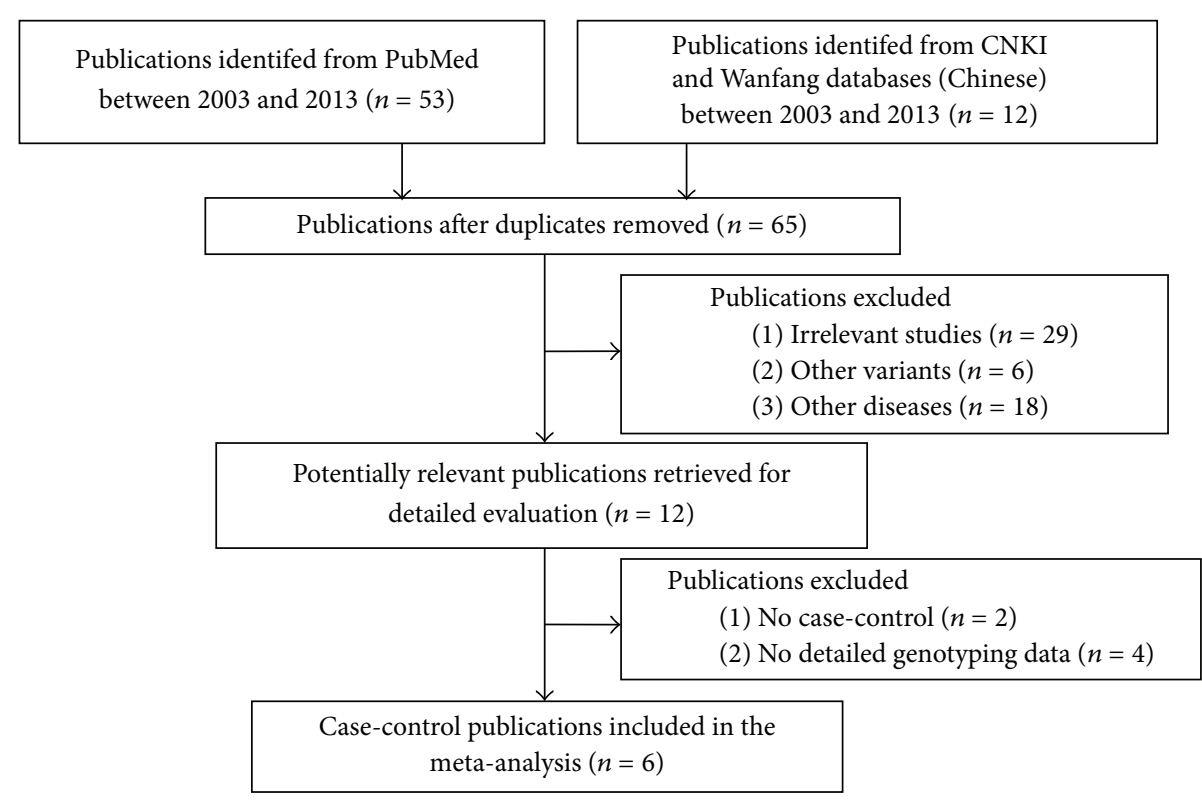

FIGURE 1: Flowing chart of selection publications in the current meta-analysis.

\section{Material and Methods}

2.1. Retrieval of Studies and Selection Criteria. We systematically search available studies from 2003 to 2013 in PubMed (English), CNKI, and Wanfang (Chinese). Keywords were "coronary heart disease" or "coronary artery disease" or "myocardial infarction" combined with "LDLR" or "low density lipoprotein receptor" or "rs2228671" and "polymorphism" or "genetic association." The inclusion criteria for the studies involved in this meta-analysis met the following criteria: (1) case-control study about LDLR rs2228671 polymorphism; (2) case-control study with genotyping or allelic information, or odd ratio (OR) with 95\% confidential interval (CI).

2.2. Data Extraction. Data included in this meta-analysis was extracted independently from all studies using the same standard protocol by two reviewers ( $\mathrm{HY}$ and $\mathrm{YH}$ ). The inclusion criteria of our meta-analysis were as follows: first author's name, publication year, ethnicity, numbers of cases and controls, genotype distribution, and OR with $95 \%$ CI.

2.3. Patients and Controls. The study protocol was approved by the ethical committee of School of Medicine, Ningbo University. A total of 162 cases and 113 controls were recruited in this study from the Affiliated Hospital of Ningbo University. All the participants in this study have signed the informed consent forms. All the 275 individuals underwent coronary angiography and were categorized into $\mathrm{CHD}$ patients and non-CHD controls according to our previous descriptions $[5,19]$. All the participants enrolled in this study were Han Chinese residing in or near Ningbo city. None of individuals in this study had congenital heart disease, cardiomyopathy and severe liver, or kidney disease.
2.4. SNP Genotyping. Genomic DNA was isolated from peripheral blood lymphocytes using standard phenolchloroform method and then was stored in TE buffer. All DNA samples were amplified by polymerase chain reaction (PCR). PCR was denatured at $94^{\circ} \mathrm{C}$ for $15 \mathrm{~s}$, followed by 45 cycles of denaturation at $94^{\circ} \mathrm{C}$ for $20 \mathrm{~s}$, annealing for $30 \mathrm{~s}$ at $56^{\circ} \mathrm{C}$, extension at $72^{\circ} \mathrm{C}$ for $1 \mathrm{~min}$, and a final extension at $72^{\circ} \mathrm{C}$ for $3 \mathrm{~min}$. DNA amplification and genotyping was performed on the SEQUENOM Mass-ARRAY iPLEX platform according to the manufacturer's instructions [5].

2.5. Statistical Analyses. Hardy-Weinberg equilibrium (HWE) was examined by the Arlequin program (version 3.5) [20]. The differences in the genotype and allele frequencies between cases and controls were analyzed by the CLUMP22 software with 10,000 Monte Carlo simulations [21]. Power analysis was performed by Power and Sample Size Calculation software [22]. Meta-analysis was made by REVMAN 5.0 (Cochrane Collaboration, Oxford, United Kingdom) and Strata 11.0 software (Strata Corporation, College Station, TX) $[23,24]$. Publication bias was evaluated by Begg and Egger regression tests [25]. The combined ORs with $95 \%$ CI values were calculated by either fixed-effect or random-effect method [26]. A two-tailed $P$ value of 0.05 or lower was defined to be statistically significant.

\section{Results}

We systematically searched in PubMed, CNKI, and Wanfang from 2003 to 2013, and selected a total of 57 literatures after removing the duplicated publications (Figure 1). According to the descriptions in the titles and abstracts, we excluded 26 irrelevant literatures, 6 literatures on other variants, and 12 literatures on other diseases. In addition, 1 literature without sufficient case-control genotyping data and 5 literatures 
TABLE 1: Characteristics of the association studies between rs2228671 and CHD.

\begin{tabular}{|c|c|c|c|c|}
\hline \multirow{2}{*}{ Author and year } & \multirow{2}{*}{ Ethnic group } & \multicolumn{2}{|c|}{ Genotype (CC/CT/TT) } & \multirow{2}{*}{$P$-allele } \\
\hline & & Cases & Controls & \\
\hline Ortlepp et al. (2003) [11] & German & $937 / 216 / 10$ & $972 / 255 / 22$ & 0.0453 \\
\hline Krawczak et al. (2006) [12] & German & $1755 / 379 / 19$ & $1840 / 474 / 25$ & 0.0184 \\
\hline Samani et al. (2007) [13] & German & $781 / 93 / 1$ & $1417 / 224 / 3$ & 0.0281 \\
\hline Samani et al. (2007) [13] & British & $1578 / 322 / 13$ & $2332 / 569 / 34$ & 0.0051 \\
\hline Schunkert et al. (2008) [14] & German & $236 / 43 / 2$ & $224 / 61 / 5$ & 0.0343 \\
\hline Erdmann et al. (2009) [15] & German & $282 / 64 / 3$ & $671 / 164 / 15$ & 0.3333 \\
\hline Martinelli et al. (2010) [16] & Italian & $549 / 134 / 9$ & $227 / 61 / 3$ & 0.73 \\
\hline Our study (2013) & Chinese & $157 / 4 / 1$ & $111 / 2 / 0$ & 0.485 \\
\hline
\end{tabular}

TABLE 2: Genotype and allele frequency distributionof $L D L R$ gene rs 2228671 polymorphism in cases and controls* .

\begin{tabular}{|c|c|c|c|c|c|c|c|c|}
\hline Gender & Group & $\mathrm{CC} / \mathrm{CT} / \mathrm{TT}$ & $\chi^{2}$ & $P(\mathrm{df}=2)$ & $\mathrm{C} / \mathrm{T}$ & $x^{2}$ & $P(\mathrm{df}=1)$ & OR (95\% CI) \\
\hline \multirow{2}{*}{ All } & Cases & $157 / 4 / 1$ & \multirow{2}{*}{0.86} & \multirow{2}{*}{1} & $318 / 6$ & \multirow{2}{*}{0.87} & \multirow{2}{*}{0.49} & \multirow{2}{*}{$0.47(0.09-2.36)$} \\
\hline & Controls & $111 / 2 / 0$ & & & $224 / 2$ & & & \\
\hline \multirow{2}{*}{ Male } & Cases & $113 / 2 / 1$ & \multirow{2}{*}{0.51} & \multirow{2}{*}{0.77} & $228 / 4$ & \multirow{2}{*}{ NA } & \multirow{2}{*}{ NA } & \multirow{2}{*}{$0.49(0.05-4.41)$} \\
\hline & Controls & $58 / 1 / 0$ & & & $117 / 1$ & & & \\
\hline \multirow{2}{*}{ Female } & Cases & $44 / 2 / 0$ & \multirow{2}{*}{0.53} & \multirow{2}{*}{0.76} & $90 / 2$ & \multirow{2}{*}{ NA } & \multirow{2}{*}{ NA } & \multirow{2}{*}{$0.42(0.04-4.71)$} \\
\hline & Controls & $53 / 1 / 0$ & & & $107 / 1$ & & & \\
\hline
\end{tabular}

${ }^{*}$ NA represents not analyzed; rs2228671 meets HWE in all groups $(P>0.05)$.

TABLE 3: Genotype and allele frequency distributionof $L D L R$ gene rs2228671 polymorphism in European population.

\begin{tabular}{lcccccccc}
\hline Gender & Group & CC/CT/TT & $\chi^{2}$ & $P(\mathrm{df}=2)$ & $\mathrm{C} / \mathrm{T}$ & $\chi^{2}$ & $P(\mathrm{df}=1)$ & $\mathrm{OR}(95 \% \mathrm{CI})$ \\
\hline \multirow{2}{*}{ European population } & Cases & $6218 / 1251 / 57$ & 20.59 & $<.0001$ & $13687 / 1365$ & 20.26 & $<.0001$ & $1.180(1.098-1.269)$ \\
& Controls & $7685 / 1808 / 107$ & & & $17178 / 2022$ & & \\
\hline
\end{tabular}

without detailed SNP information were also removed. At last, 6 literatures [11-16] on 7 case-control studies were harvested in our meta-analysis (Table 1). Furthermore, we performed a case-control study in Han Chinese population, and it was later included in our meta-analysis.

Genotype distribution of rs2228671 in our case-control study met HWE for both CHD cases and non-CHD controls $(P>0.05)$, indicating that our case-control study had a well-characterized random sampling. Our case-control study suggested that LDLR rs2228671-T allele was rare in Chinese population (cases: $2 \%$; controls: $1 \%$ ), and this agrees with the frequency in HapMap Chinese Han in Beijing (CHB) population (0-2\%). No significant difference in the genotype distribution between $\mathrm{CHD}$ cases and non-CHD controls are revealed in all samples $(P>0.05$; Table 2$)$ and in the subgroup analysis by gender $(P>0.05$; Table 2$)$. In summary, our case-control study showed that there was no association between LDLR rs2228671 and CHD in Chinese. However, significant association was found between $L D L R$ rs2228671 and CHD in European population $\left(\chi^{2}=20.59\right.$, $P<0.0001$ by genotype; $\chi^{2}=20.26$; OR $=1.180,95 \%$ CI $=1.098-1.269, P<0.0001$ by allele; Table 3$)$. Using the fixed-effect method, our meta-analysis contained 7,588 CHD patients and 9,711 controls from German, British, Italian, and Chinese populations. As shown in Figure 2, significant association was observed between rs2228671 and CHD $(P=$ $0.0005, \mathrm{OR}=0.83$, and $95 \% \mathrm{CI}=0.75-0.92)$. In addition, no heterogeneity among the studies was included in this metaanalysis ( $I^{2}=0 \%$; Figure 2$)$. Furthermore, no obvious visual evidence of publication bias in the meta-analysis was shown by funnel plot $(P>0.05$; Figure 3$)$.

\section{Discussion}

Aberrant LDLR level in blood can cause abnormal cholesterol metabolism [2]. As the main pathogenic gene of FH, LDLR gene is associated with multiple vascular diseases $[15,16,27]$. Polymorphisms of LDLR gene were associated with type 2 diabetes [28] and hypertension [29] that also related to CHD. Recently, a handful of LDLR polymorphisms have been studied in CHD, including those (rs14158, rs3826810, rs1433099, rs2738464, rs2738465, and rs2738466) in the $3^{\prime}$ untranslated region ( $3^{\prime}-$ UTR) and rs2228671 in second exon [30-32]. SNPs in first intron (rs6511720) and $5^{\prime}$ flanking region (rs17248720) of $L D L R$ gene were closely related to both LDL-C and CHD $[33,34]$. Rs1433099 and rs2738466 in the $3^{\prime}$ UTR of LDLR were reported to be associated with baseline lipids in American population [32]. The T allele of rs2228671 polymorphism was associated with higher FVIII:c levels. In addition, LDLR rs2228671 may be regulated FVIII:c levels 


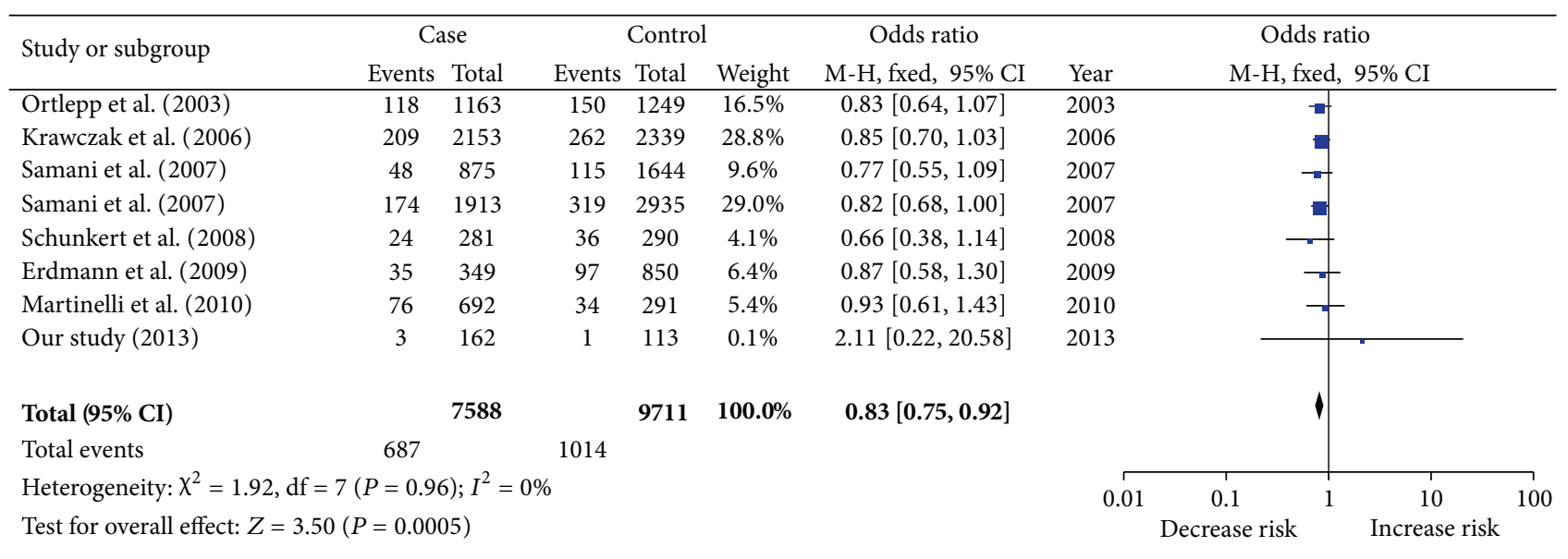

FIGURE 2: Meta-analysis of rs2228671 with CHD.

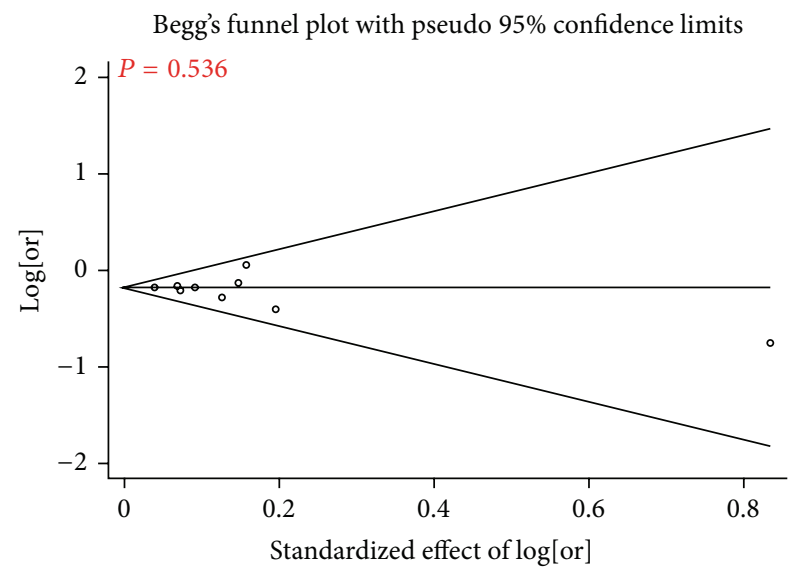

(a)

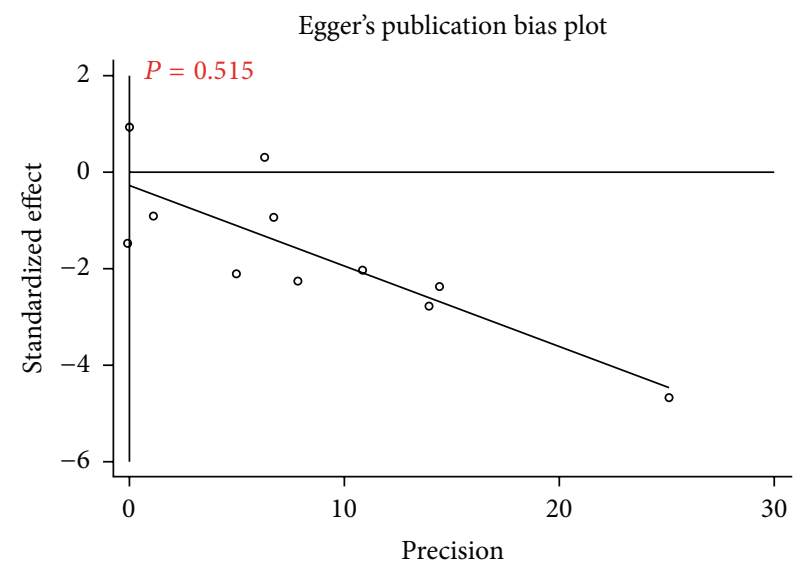

(b)

FIgURE 3: Publication bias analysis of 8 studies in the meta-analysis. The Begg's funnel plot and the Egger's publication bias plot test also indicated little evidence of publication bias among studies of rs2228671 and CHD risk (Begg, $P=0.536$; Egger, $P=0.515$ ).

and associated with the independence risk factor (plasma lipids) of CHD [16].

Our meta-analysis among 17299 individuals showed that rs2228671-T allele reduced the risk of coronary heart disease in the combined samples from German, British, Italian, and Chinese populations ( $\mathrm{OR}=0.83, P=0.0005)$. Furthermore, rs2228671-T allele frequencies in the meta-analysis among German, British, and Italian populations were 7$12.2 \%$ that is similar to $10 \%$ in HapMap CEU population. However, rs2228671-T allele frequency is 0\% in HapMap CHB population and $0.9 \%$ in the controls of our study. Due to the rare allele of $L D L R$ rs 2228671 in our samples, the power of our case-control study was only $5.1 \%$, in contrast of $100 \%$ in the present meta-analysis. This suggests that a lack of association in our case-control study may largely be explained by the insufficient power for this rare polymorphism and the small sample size. Future investigation on other common $L D L R$ polymorphisms is worth being performed in a large Chinese cohort.
Human $L D L R$ is about $43 \mathrm{~kb}$ in length and has 1367 active polymorphism. As shown in our study, the allele frequency of rs1122608-T is much lower than those in the European studies; suggesting a cross-population comparison of this polymorphism may help one understand the role of $L D L R$ in different ethnic population. Meanwhile, the previous tested LDLR rs1122608 polymorphism did not yield a significant result $(P=0.148)$ [35], in contrast to a significant result of rs2228671 in the current study $(P=0.0005)$. This suggests rs2228671 and rs1122608 might exert different contributions to the risk of CHD.

There were several limitations in our study as follows. Firstly, most of the involved individuals in our meta-analysis were Europeans; thus our result might not be applied to other populations such as Chinese. Secondly, although we had no evidence of the publication bias in our meta-analysis, we cannot exclude the possibility of existing potential bias upon reporting the studies without significant association results. Last but not least, the power of our case-control study in 
Chinese is only $5.1 \%$. The negative result of rs2228671 might not represent for other variants of $L D L R$ gene in Chinese population.

In conclusion, the meta-analysis demonstrated that the $L D L R$ rs2228671-T allele is a protective factor of CHD in Europeans. However, the case-control study showed no significant association of LDLR rs2228671 with CHD in Han Chinese population.

\section{Conflict of Interests}

The authors declare no conflict of interests.

\section{Authors' Contribution}

Huadan Ye, Qianlei Zhao and Yi Huang are co-first authors of this work.

\section{Acknowledgments}

The research was supported by the grants from National Natural Science Foundation of China (31100919 and 81371469), Natural Science Foundation of Zhejiang Province (LR13H020003), K. C. Wong Magna Fund in Ningbo University, Natural Science Foundation of Ningbo City (2011A610037), and Ningbo Social Development Research Projects (2012C50032).

\section{References}

[1] A. A. Phillips, A. T. Cote, S. S. Bredin, and D. E. Warburton, "Heart disease and left ventricular rotation-a systematic review and quantitative summary," BMC Cardiovascular Disorders, vol. 12, article 46, 2012.

[2] M. S. Brown and J. L. Goldstein, "Expression of the familial hypercholesterolemia gene in heterozygotes: mechanism for a dominant disorder in man," Science, vol. 185, no. 4145, pp. 6163, 1974.

[3] I. Ejarque, J. T. Real, S. Martinez-Hervas et al., "Evaluation of clinical diagnosis criteria of familial ligand defective apoB 100 and lipoprotein phenotype comparison between LDL receptor gene mutations affecting ligand-binding domain and the R3500Q mutation of the apoB gene in patients from a South European population," Translational Research, vol. 151, no. 3, pp. 162-167, 2008.

[4] E. W. Lee, M. Michalkiewicz, J. Kitlinska et al., "Neuropeptide $\mathrm{Y}$ induces ischemic angiogenesis and restores function of ischemic skeletal muscles," Journal of Clinical Investigation, vol. 111, no. 12, pp. 1853-1862, 2003.

[5] J. Zhou, Y. Huang, R. S. Huang et al., "A case-control study provides evidence of association for a common SNP rs974819 in PDGFD to coronary heart disease and suggests a sex-dependent effect," Thrombosis Research, vol. 130, pp. 602-606, 2012.

[6] R. Bulbulia and J. Armitage, "LDL cholesterol targets-how low to go?" Current Opinion in Lipidology, vol. 23, pp. 265-270, 2012.

[7] R. Alonso, N. Mata, S. Castillo et al., "Cardiovascular disease in familial hypercholesterolaemia: Influence of low-density lipoprotein receptor mutation type and classic risk factors," Atherosclerosis, vol. 200, no. 2, pp. 315-321, 2008.
[8] S. Kathiresan, C. J. Willer, G. M. Peloso et al., "Common variants at 30 loci contribute to polygenic dyslipidemia," Nature Genetics, vol. 41, no. 1, pp. 56-65, 2009.

[9] S. Kathiresan, B. F. Voight, S. Purcell et al., "Genome-wide association of early-onset myocardial infarction with single nucleotide polymorphisms and copy number variants," Nature Genetics, vol. 41, no. 3, pp. 334-341, 2009.

[10] P. Linsel-Nitschke, A. Götz, J. Erdmann et al., "Lifelong reduction of LDL-cholesterol related to a common varriant in the LDL-receptor gene decreases the risk of coronary artery disease-a Mendelian randomisation study," PLoS ONE, vol. 3, no. 8, Article ID e2986, 2008.

[11] J. R. Ortlepp, A. Von Korff, P. Hanrath, K. Zerres, and R. Hoffmann, "Vitamin D receptor gene polymorphism BsmI is not associated with the prevalence and severity of CAD in a largescale angiographic cohort of 3441 patients," European Journal of Clinical Investigation, vol. 33, no. 2, pp. 106-109, 2003.

[12] M. Krawczak, S. Nikolaus, H. Von Eberstein, P. J. Croucher, N. E. El Mokhtari, and S. Schreiber, "PopGen: Population-based recruitment of patients and controls for the analysis of complex genotype-phenotype relationships," Community Genetics, vol. 9, no. 1, pp. 55-61, 2006.

[13] N. J. Samani, J. Erdmann, A. S. Hall et al., "Genomewide association analysis of coronary artery disease," New England Journal of Medicine, vol. 357, no. 5, pp. 443-453, 2007.

[14] H. Schunkert, A. Götz, P. Braund et al., "Repeated replication and a prospective meta-analysis of the association between chromosome 9p21.3 and coronary artery disease," Circulation, vol. 117, no. 13, pp. 1675-1684, 2008.

[15] J. Erdmann, A. Großhennig, P. S. Braund et al., "New susceptibility locus for coronary artery disease on chromosome 3q22.3," Nature Genetics, vol. 41, no. 3, pp. 280-282, 2009.

[16] N. Martinelli, D. Girelli, B. Lunghi et al., "Polymorphisms at LDLR locus may be associated with coronary artery disease through modulation of coagulation factor VIII activity and independently from lipid profile," Blood, vol. 116, no. 25, pp. 5688-5697, 2010.

[17] S. Cassese, A. de Waha, G. Ndrepepa et al., "Intra-aortic balloon counterpulsation in patients with acute myocardial infarction without cardiogenic shock. A meta-analysis of randomized trials," American Heart Journal, vol. 164, article e51, pp. 58-65, 2012.

[18] C. Wang, T. Sun, H. Li, J. Bai, and Y. Li, "Lipoprotein lipase Ser447Ter polymorphism associated with the risk of ischemic stroke: a meta-analysis," Thrombosis Research, vol. 128, no. 5, pp. e107-e112, 2011.

[19] E. Rapaport, R. Bernard, and E. Corday, "Nomenclature and criteria for diagnosis of ischemic heart disease. Report of the Joint International Society and Federation of Cardiology/World Health Organization Task Force on standardization of clinical nomenclature," Circulation, vol. 59, no. 3, pp. 607-609, 1979.

[20] L. Excoffier and H. E. L. Lischer, "Arlequin suite ver 3.5: a new series of programs to perform population genetics analyses under Linux and Windows," Molecular Ecology Resources, vol. 10, no. 3, pp. 564-567, 2010.

[21] P. C. Sham and D. Curtis, "Monte Carlo tests for associations between disease and alleles at highly polymorphic loci," Annals of Human Genetics, vol. 59, no. 1, pp. 97-105, 1995.

[22] W. D. Dupont and W. D. Plummer Jr., "Power and sample size calculations. A review and computer program," Controlled Clinical Trials, vol. 11, no. 2, pp. 116-128, 1990. 
[23] D. F. Stroup, J. A. Berlin, S. C. Morton et al., "Meta-analysis of observational studies in epidemiology: a proposal for reporting," Journal of the American Medical Association, vol. 283, no. 15, pp. 2008-2012, 2000.

[24] J. W. Bisson and V. J. Cabelli, "Membrane filter enumeration method for Clostridium perfringens," Applied and Environmental Microbiology, vol. 37, no. 1, pp. 55-66, 1979.

[25] M. Egger, G. Davey Smith, M. Schneider, and C. Minder, "Bias in meta-analysis detected by a simple, graphical test," British Medical Journal, vol. 315, pp. 629-634, 1997.

[26] R. DerSimonian and N. Laird, "Meta-analysis in clinical trials," Controlled Clinical Trials, vol. 7, no. 3, pp. 177-188, 1986.

[27] S. Kathiresan, O. Melander, C. Guiducci et al., "Six new loci associated with blood low-density lipoprotein cholesterol, highdensity lipoprotein cholesterol or triglycerides in humans," Nature Genetics, vol. 40, no. 11, pp. 189-197, 2008.

[28] S. H. Wu, Y. Q. Wang, and D. Q. Sun, "The association of HincII/low density lipoprotein receptor (LDLR) restriction fragment length polymorphism (RFLP) with diabetes mellitus and its lipid phenotype with PCR gene amplification," Zhonghua Yi Xue Za Zhi, vol. 73, no. 1, pp. 10-60, 1993.

[29] Y. Yamada, K. Kato, T. Yoshida et al., "Association of polymorphisms of ABCA1 and ROS1 with hypertension in Japanese individuals," International Journal of Molecular Medicine, vol. 21, no. 1, pp. 83-89, 2008.

[30] J. M. Murabito, C. C. White, M. Kavousi et al., "Association between chromosome 9p21 variants and the ankle-brachial index identified by a meta-analysis of 21 genome-wide association studies," Circulation Cardiovascular Genetics, vol. 5, pp. 100-112, 2012.

[31] W. Chen, S. Wang, Y. Ma et al., "Analysis of polymorphisms in the $3^{\prime}$ untranslated region of the LDL receptor gene and their effect on plasma cholesterol levels and drug response," International Journal of Molecular Medicine, vol. 21, no. 3, pp. 345-353, 2008.

[32] E. Polisecki, H. Muallem, N. Maeda et al., "Genetic variation at the LDL receptor and HMG-CoA reductase gene loci, lipid levels, statin response, and cardiovascular disease incidence in PROSPER," Atherosclerosis, vol. 200, no. 1, pp. 109-114, 2008.

[33] F. Takeuchi, M. Isono, T. Katsuya et al., "Association of genetic variants influencing lipid levels with coronary artery disease in Japanese individuals," PLoS ONE, vol. 7, Article ID e46385, 2012.

[34] P. Jeemon, K. Pettigrew, C. Sainsbury, D. Prabhakaran, and S. Padmanabhan, "Implications of discoveries from genome-wide association studies in current cardiovascular practice," World Journal of Cardiology, vol. 3, pp. 230-247, 2011.

[35] L. Zhang, F. Yuan, P. Liu et al., "Association between PCSK9 and LDLR gene polymorphisms with coronary heart disease: casecontrol study and meta-analysis," Clinical Biochemistry, vol. 46, pp. 727-732, 2013. 

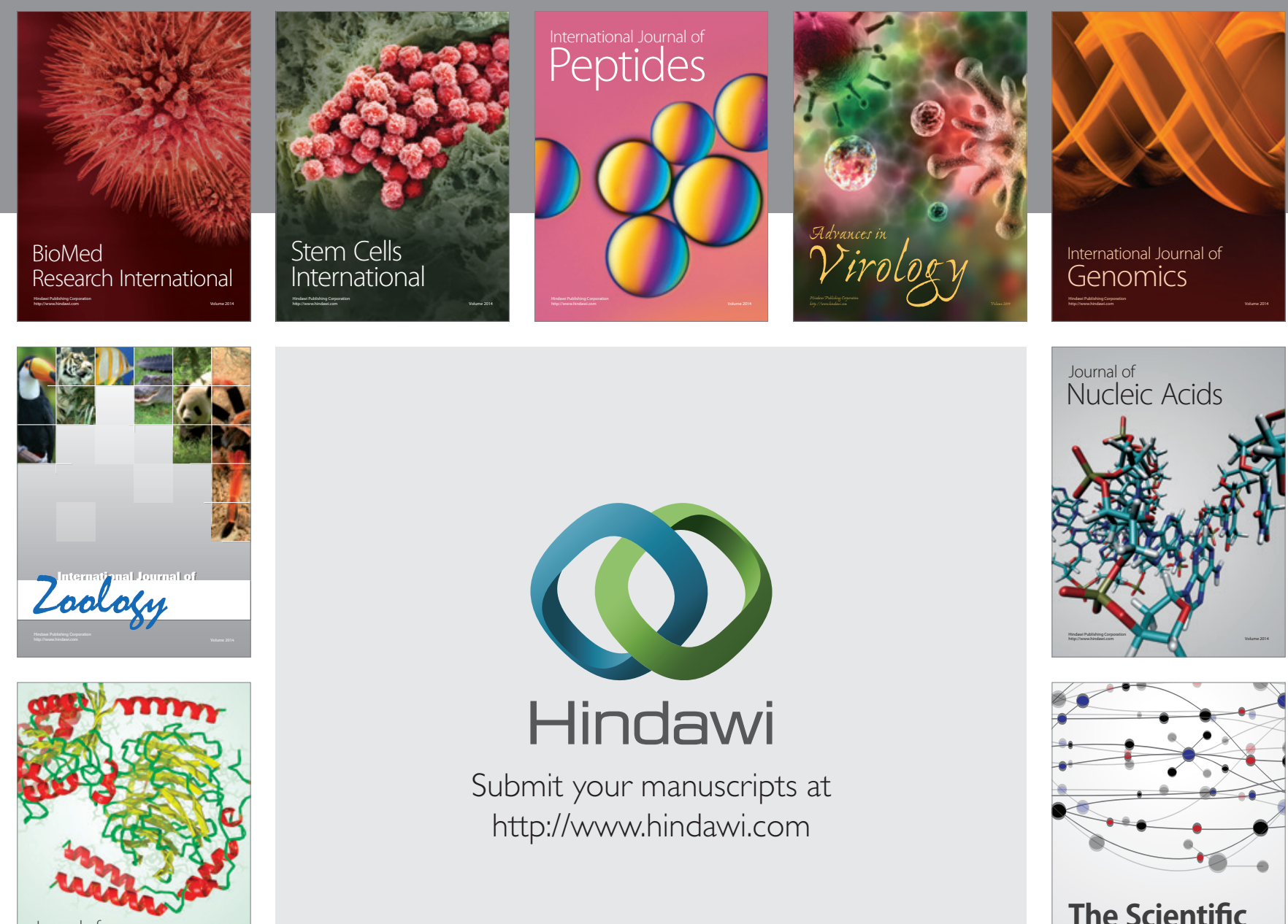

Submit your manuscripts at

http://www.hindawi.com

Journal of
Signal Transduction
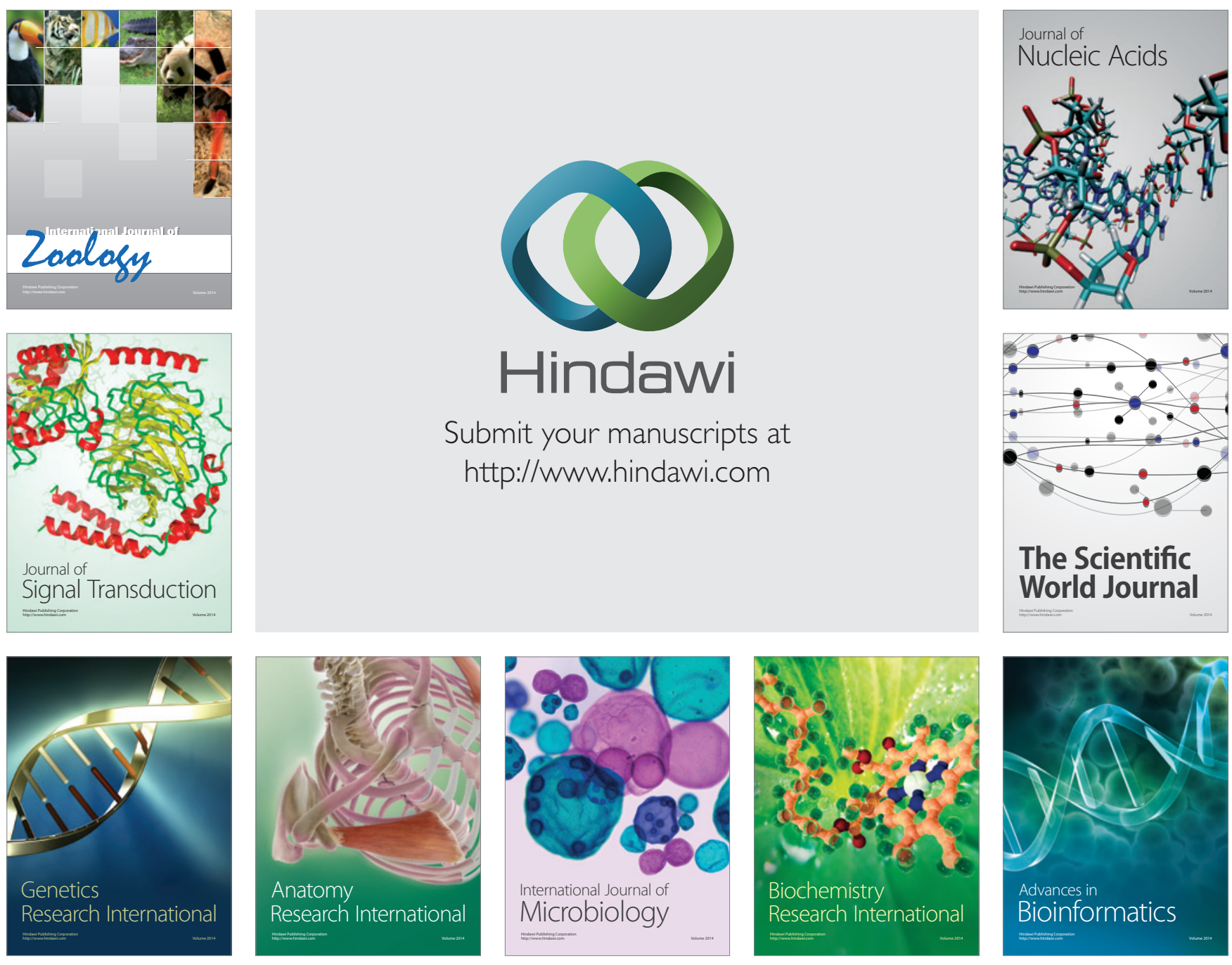

The Scientific World Journal
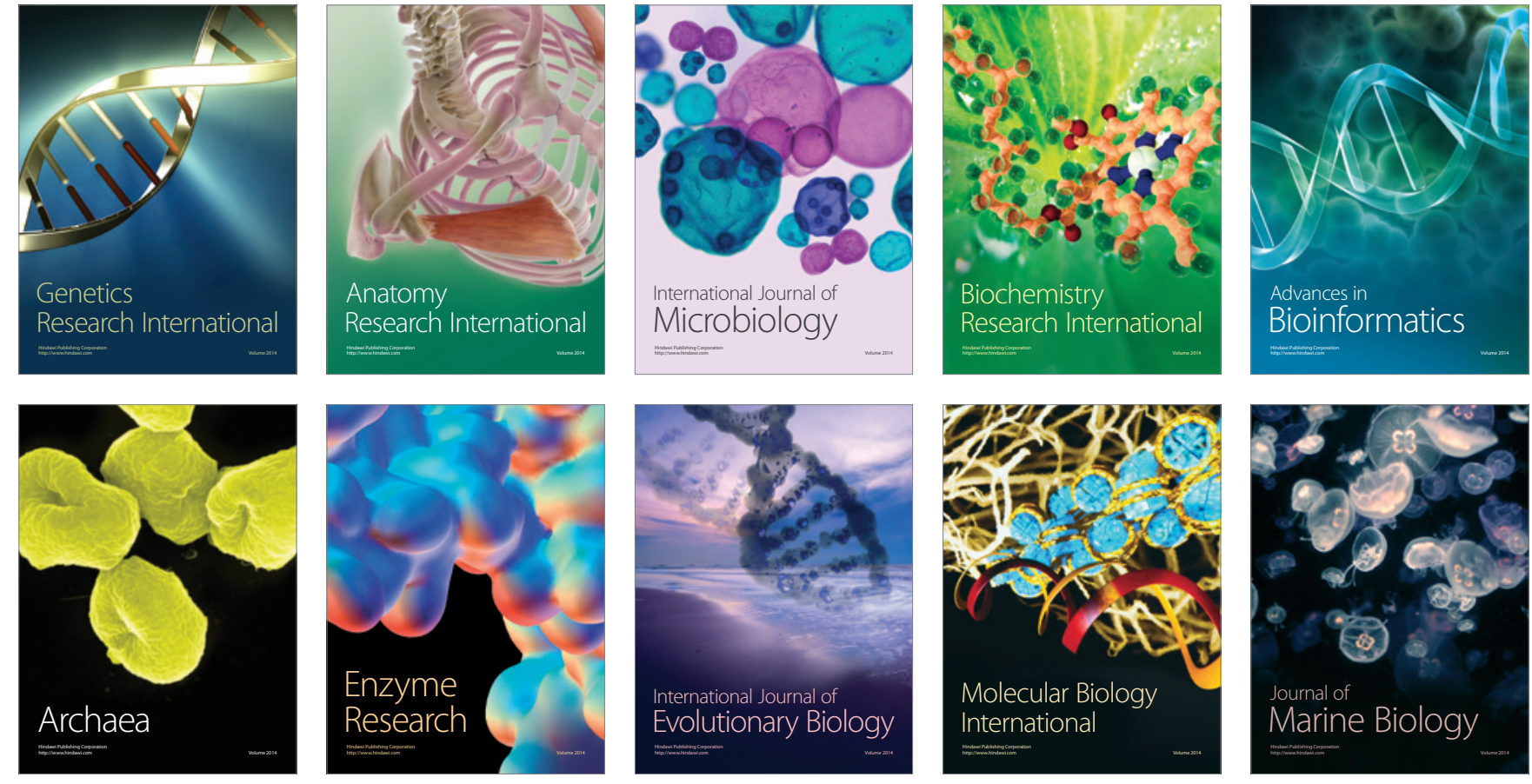